\title{
Flow Rate Estimation using Dynamic Artificial Neural Networks with Ultrasonic Level Measurements
}

\author{
Khim Chhantyal Minh Hoang Håkon Viumdal Saba Mylvaganam \\ Faculty of Technology, Natural Sciences, and Maritime Sciences, University College of Southeast Norway, \\ \{khim.chhantyal, hakon.viumdal, saba.mylvaganam\}@usn.no, m.hoang1304@gmail.com
}

\begin{abstract}
Accurate estimation of flow in drilling operations at inflow and outflow positions can lead to increased safety, optimized production and improved cost efficiency. In this paper, Dynamic Artificial Neural Network (DANN) is used to estimate the flow rate of non-Newtonian drilling fluids in an open channel Venturi-rig that may be used for estimating outflow. Flow in the Venturi-rig is estimated using ultrasonic level measurements. Simulation study looks into fully connected Recurrent Neural Network (RNN) with three different learning algorithms: Back Propagation Through Time (BPTT), Real-Time Recurrent Learning (RTRL) and Extended Kalman Filter (EKF). The simulation results show that BPTT and EKF algorithms converge very quickly as compared to RTRL. However, RTRL gives more accurate results, is less complex and computationally fastest among these three algorithms. Hence, in the experimental study RTRL is chosen as the learning algorithm for implementing Dynamic Artificial Neural Network (DANN). DANN with RTRL learning algorithm is compared with Support Vector Regression (SVR) and static ANN models to assess their performance in estimating flow rates. The comparisons show that the proposed DANN is the most accurate model among three models as it uses previous inputs and outputs for the estimation of current output.
\end{abstract}

Keywords: drilling operations, open channel venturi flume, non-Newtonian fluid, flow rate estimation, ultrasonic level measurements, recurrent neural network, realtime recurrent learning

\section{Introduction}

In drilling operations, the drilling mud is circulated in a closed loop starting from the mud tank into the wellbore and back to the mud tank. The mud can be water-based, oil-based or gas-based and is circulated during the drilling operation, until the desired depth is reached. During circulation, the rheological properties of drilling mud have significant importance for the safe and efficient drilling operation. The viscosity, density, and flow rate of circulating mud play a vital role, in all the drilling operations. (Caenn et al., 2011)

In general, drilling muds are non-Newtonian in nature, and the viscosity of the mud along with other rheologi- cal properties govern the transport of rock cuttings while drilling. (Caenn et al., 2011)

The density or mud weight is mainly responsible for maintaining the pressure in the wellbore. Depending on the types of the drilling operation and the reservoir, the wellbore pressure or bottom-hole pressure $\left(P_{b}\right)$ is limited within the pressure window given by formation pressure $\left(P_{f}\right)$ and formation fracture pressure $\left(P_{f f}\right)$. If the wellbore pressure is less than the formation pressure $\left(P_{b}<P_{f}\right)$, the formation gasses and fluids will flow into the drilling mud, and is called kick. The occurrences of kick should be detected as early as possible during drilling operations. If the early kick detection is ignored or is not detected, it can lead to problems in maintaining the density of the mud and in the extreme case, it can result in blow-out of hydrocarbons on the rig, e.g. the Deepwater Horizon explosion, (Hauge and Øien, 2012). In the case of $\left(P_{b}>P_{f}\right)$, the high pressure circulating fluids may enter the formation pores, causing fluid losses. If the wellbore pressure is further increased, beyond the formation fracture pressure $\left(P_{b}>P_{f f}\right)$, the circulation fluid can fracture the formation and cause an increased fluid loss, often called lost circulation. The fluid loss will decrease the volume of the mud in the circulation loop and in the mud tank, and will affect the production, (Caenn et al., 2011).

A similar situation occurs frequently in geothermal drilling. In geothermal drilling, one of the costly problems is lost circulation. that occurs when drilling fluid is lost to the formation rather than returning to the surface, preferably intact. The management of lost circulation is important and requires the accurate measurement of drilling fluid flow rate both into and out of the well.

Reliable detection of unusual conditions can allow the use of low weight mud, efficient drilling, less formation damage, and lead to lower drilling costs. Delta flow method, i.e. calculating the difference between flows at inflow and outflow points of the circulation mud, is one of the best methods to detect kick and fluid loss, which uses the flow measurements before and after the wellbore, (Maus et al., 1979; Speers et al., 1987; Orban et al., 1987, 1988; Schafer et al., 1991; Kamyab et al., 2010). The difference in outflow and inflow measurements can be used as an indication of unusual conditions while drilling. If the flow rate before wellbore is less than the flow rate in the return line, then it can be considered as an indication of 
early kick detection. Whereas, if the inflow is greater than the outflow, it is an early indication of fluid loss. In addition, the flow rate of circulating fluid will determine the transportation of rock cuttings. The flow velocity of the circulation mud is often maintained higher than the settling velocity of the rock cuttings for efficient transportation of cuttings. In addition to the delta flow method, other methods of early kick detection are discussed in (Kamyab et al., 2010; Mills et al., 2012; Ali et al., 2013; Patel et al., 2013; Vajargah et al., 2013).

In literature (Maus et al., 1979; Speers et al., 1987; Orban et al., 1987, 1988; Schafer et al., 1991; Kamyab et al., 2010), there are different systems for measuring delta flow. For inflow measurement, conventional pump stroke counter, rotatory pump speed counter, magnetic flow meter, Doppler ultrasonic flow meter or Coriolis mass flowmeter can be used. For outflow measurement, magnetic flowmeter, Doppler ultrasonic-based flowmeter, standard paddle meter, ultrasonic level meter, a prototype rolling float meter or open channel Venturi flowmeter can be used. The scenario of inflow and outflow measurement is completely different. For example, the inflow measurement can be carried out using Coriolis mass flow meter, more accurate but an expensive flowmeter. However, Coriolis mass flow meter is not suitable for outflow measurements as the returning mud contains solid rock cuttings, other formation particles, formation fluids and gases. An overview of different flowmeters based on reliability and accuracy is given in (Schafer et al., 1991). Based on this analysis, magnetic flowmeter or Doppler ultrasonic flowmeter are suitable for inflow measurements and prototype rolling float meters for outflow measurement. (Speers et al., 1987) presents the implementation of delta flow method by using magnetic flowmeters at inflow and outflow locations. The magnetic flowmeter is limited in applications to conductive fluids or to only water-based muds. In addition, magnetic flowmeters need some additional U-tube design in the return section. For lower flow velocity of circulating fluids, the rock cuttings will settle at the bottom of this U-tube. These problems are avoided in open channel return line, in which efficient rock cutting transportation and their easier separation from mud, (Orban et al., 1987, 1988).

This paper presents the outflow measurement based on open channel flow with a Venturi section. In an open channel flow, the upstream pressure relative to a reference level in the control section of the loop structure can be used to estimate the flow rate, (White, 2002). The control section used in the flow loop is the Venturi flume. The flow measurement is based on an extension of the application of the well-known Venturi principle, to flow of fluid in an open channel, (Skorpik, 2013). The constriction in the Venturi section results in the transition of flow from subcritical to supercritical flow in the vicinity of the throat, (Frenzel, 2011). For sufficiently long throat, the critical condition occurs in the throat, giving the critical depth, (Geratebau, 2013). The level of the fluid in upstream is measured as the critical depth is identified. The level can be measured using ultrasonic or RADAR level sensors and flow rate can be calculated as a function of measured level.

To study the possibility of using Venturi flume in estimating flow rate, a flow loop (i.e. Venturi rig) is available in University College of Southeast Norway (USN), Porsgrunn, Norway. For this Venturi rig, the CFD simulation study of open channel flow measurement is investigated in (Berg et al., 2013). The numerical algorithm using Saint Venant equation is presented in (Agu and Lie, 2014a,b). However, the developed numerical model is not applicable for real-time monitoring and controlling purpose due to the high computational cost. The study presented in (Chhantyal et al., 2016b) shows the successful implementation of static Artificial Neural Network (ANN) and Support Vector Regression (SVR) techniques for flow measurement in the test loop. The present study is a continuation, where, Dynamic Artificial Neural Networks (DANN) are investigated and implemented in the software used in running, monitoring and controlling the flow loop.

In the following sections, the simulation study of fully connected Recurrent Neural Network (RNN) with three different learning algorithms for estimating the flow rate of the non-Newtonian liquids is presented. Finally, the experimental results of flow rate estimation using RNN, ANN and SVR are discussed.

\section{Dynamic Artificial Neural Network}

ANN can be of the static or dynamic type. Static ANN or feedforward ANN type uses current inputs and current outputs whereas, DANN uses current and previous inputs and outputs for modeling purpose. Further, DANN can be partially connected RNN or fully connected RNN based on the feedback loops. Fully connected RNNs have selffeedback loops, and partially connected RNNs does not have self-feedback loops (Dijk, 1999). Some useful details of the MATLAB Toolbox DANN are given in the companion paper in this volume (Chhantyal et al., 2016a).

The delta flow measurement discussed in Section 1 is a dynamic problem, where the previous information about the kick detection and fluid loss is important for the current measurement. Therefore, fully connected RNN is used for modeling, the estimation of the flow rate being based on the level measurements in the open channel Venturi flow loop. For the estimation of the flow rate, three different learning algorithms are used. These algorithms are presented here briefly.

\subsection{Back Propagation Through Time (BPTT)}

BPTT is an extension of gradient-based back propagation algorithm that is used in static ANN. The idea in BPTT is to unfold the RNN architecture into feedforward ANN architecture in an arbitrary number of time steps or folds. These folds make the error to propagate even further in time, so it is called back propagation through time. However, the number of folds are usually low to avoid deep network and this approach is called is often called trun- 


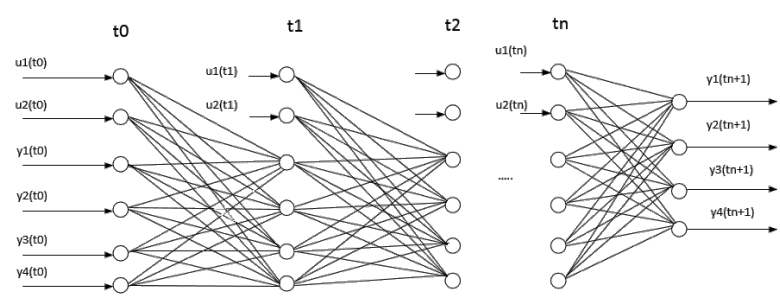

Figure 1. A general architecture for Back Propagation Through Time (BPTT) learning algorithm with $\mathrm{N}$ number of neurons and $\mathrm{n}$ numbers of foldings.

cated BPTT. In general, recurrent weights are simply duplicated over the folds while unfolding, (Boden, 2001). The basic BPTT architecture is shown in Figure 1. The computational complexity of BPTT is of order $O\left(N^{2}\right)$ and the storage requirement is of order $O\left(N^{2}\right)$, where $\mathrm{N}$ being number of neurons and $\mathrm{n}$ is the arbitrary number of folds. The drawbacks of BPTT are; it is an offline learning algorithm and requires large memory to store state information at different folds (Williams, 1992).

\subsection{Real Time Recurrent Learning (RTRL)}

RTRL is one of the most used real-time learning algorithms for RNN. In RTRL, the gradients at timet are computed based on the gradients at previous time steps. The gradient information is propagated in time (Mak et al., 1999; Mandic and Chambers, 2000; Budik, 2006). The basic RTRL architecture is shown in Figure 2. The connections with blue color are the additional self-feedback and feedback connections, which is not included in static ANN. These additional connections make the network get previous input values and output values and consider them as additional internal inputs in the current time. By doing this, a network can work dynamically. However, RTRL algorithm suffers from slow convergence, which is typical for all gradient-based algorithms. Mandic and Chambers, (Mandic and Chambers, 2000) has presented an RTRLbased learning algorithm with an adaptive learning rate that can improve the convergence performance. RTRL further suffers from the large computational complexity of the order of $O\left(N^{4}\right)$ and even critically with a storage requirement of the order of $O\left(N^{3}\right)$, (Williams, 1992).

\subsection{Extended Kalman Filter Learning (EKF)}

EKF is a recursive algorithm that computes state estimations based on the previous state information at the cur-

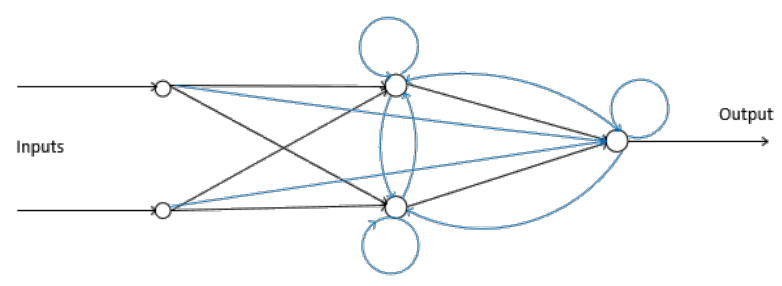

Figure 2. A general architecture for Recurrent Neural Network (RNN) with self-feedback and feedback loops from neurons. rent time, (Kim, 2011). EKF can be used as a supervised on-line learning algorithm to determine the weights of an RNN. In EKF learning algorithm, the state vector consists of weights and the locally induced outputs of each neuron in the network. Regarding convergence to a solution, EKF is very fast compared to BPTT and RTRL. The order of computational complexity for EKF is same as RTRL, $O\left(N^{4}\right)$, and the storage requirement increases to the order of $O\left(N^{4}\right)$ for EKF. The RTRL algorithm is identical to the simplified EKF algorithm, and the architecture is the same, (Williams, 1992).

\section{Experimental Set-up}

To develop RNN models, model-drilling fluid is circulated in the flow loop. The circulated fluid is visco-plastic in nature with the fluid properties of density at $1136 \mathrm{~kg} / \mathrm{m} 3$ and a viscosity ranging from $23-180$ [centipoise] for the $500-1\left[s^{-1}\right]$ shear rate. Figure 3 shows the open channel section of flow rig with a Venturi constriction and three ultrasonic level sensors. The mass flow measurement is performed using Coriolis mass flow meter and is considered as a reference for RNN models. Recent study shows that the level measurements at the throat (LT18), the level of the downstream (LT-17) and the level of the upstream (LT-15) are highly correlated to flow rate (Chhantyal et al., 2016b). Therefore, these variables are considered for modeling and are given in Table 1 and some concurrent measurements from these three ultrasonic sensors are shown in Figure 4, along with simultaneous measurements of flow from a Coriolis meter.

For the mass flow rate range of $250-500[\mathrm{~kg} / \mathrm{min}], 1800$ data samples for each variable are measured. The data samples are normalized in the range of (0-1). Out of 1800 normalized data samples, $70 \%, 15 \%$ and $15 \%$ of data are selected as training, validation, and test sets respectively.

\section{Results}

This paper presents results from both simulations based on the three models and practical implementation of RNN for flow rate measurement in an open channel flow loop.

\subsection{Simulation Study}

RNN is implemented using all the three learning algorithms discussed in Section 2. Table 2 shows the optimal parameters used in the simulations. These optimal parameters are determined using grid search method and the optimization is done using Mean Absolute Percentage Error (MAPE). Apart from these parameters, number of neurons selected is 7, learning rate is 0.1 and number of folds for BPTT is 7.

Figure 5 shows the comparison of RNN with different algorithms. As discussed in Section 2, EKF learning algorithm can quickly converge to a solution. From Figure 5 showing the MSE, it can be seen that EKF converges well before 20 epochs, BPTT converges around 100 epochs, and RTRL takes around 300 epochs to converge. The con- 
Table 1. Input and output variables used for developing RNN models with the range and variable type.

\begin{tabular}{lccc}
\hline Variables & Range & Units & Type \\
\hline Upstream level measurement & $31.2-107.5$ & $\mathrm{~mm}$ & Input \\
Level measurement at the throat & $28.9-78.3$ & $\mathrm{~mm}$ & Input \\
Downstream level measurement & 44.3106 .6 & $\mathrm{~mm}$ & Input \\
Mass flow rate & $250-500$ & $\mathrm{~kg} / \mathrm{min}$ & Output \\
\hline
\end{tabular}

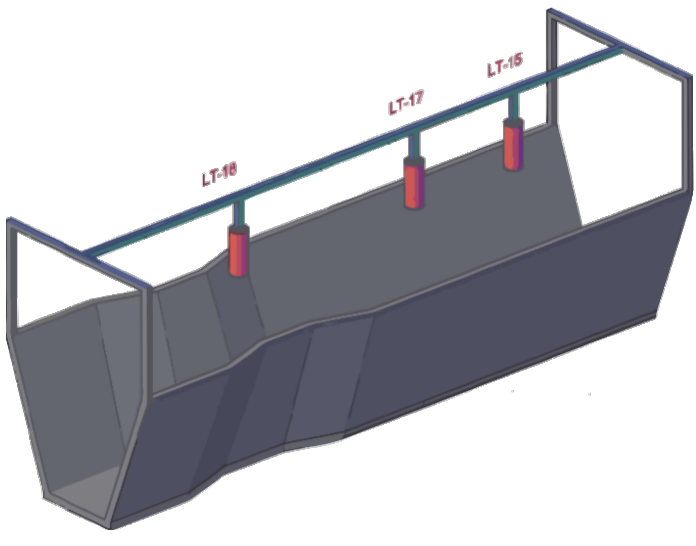

(a)

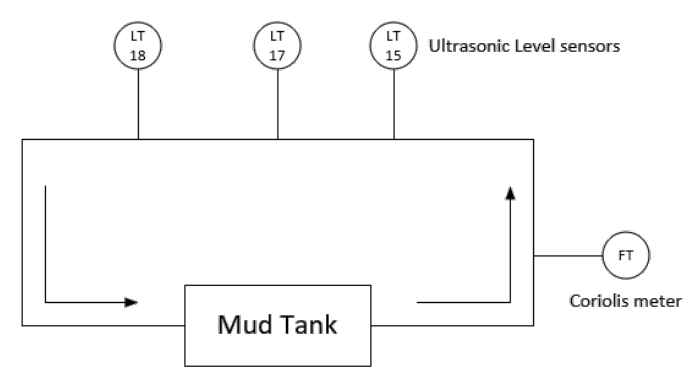

(b)

Figure 3. a) An open channel with Venturi section and three level sensors, LT-15, LT-17 and LT-18, with an arrow showing a flow direction. (b) Extremely simplified P\&ID for the Venturirig flow loop with the measurands used in this study, viz. ultrasonic level sensors and FT-Coriolis mass flowmeter.

verging efficiency of these algorithms can be observed using the state parameters, which are weights of the neural network. Figure 6 shows the states of some of the weights while training a network. The state representation shows that the states in EKF and BPTT algorithms go to steady state very quickly. However, RTRL needs numerous training epochs for achieving steady states.

Figure 7 shows the estimations of different learning algorithms with reference to flow measurements from Coriolis mass flowmeter. The simulation results show that all the models using different learning algorithms are capable of describing the dynamics of the reference flow measurements well. RTRL has minimum MAPE out of the three models used, as shown in Table 2.
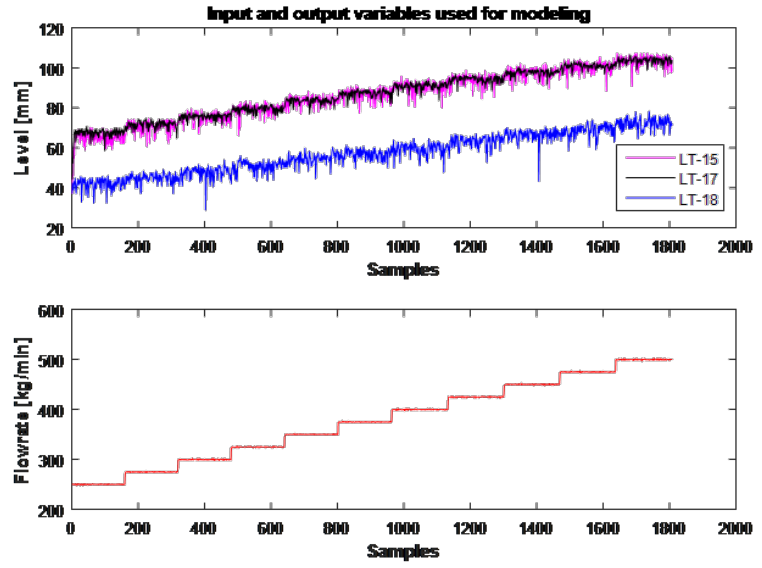

Figure 4. Input and output variables used for developing RNN models. First plot shows three level measurements with LT-15, LT-17 and LT-18. Second plot shows flowrate measurement using Coriolis mass flowmeter.

\subsection{Experimental Study}

The experimental study involves the implementation of simulation study in the Venturi rig. Despite slow convergence, RNN with RTRL learning algorithm is selected for its accuracy, less complexity, and faster computation. The algorithms for both BPTT and EKF have complex architectures and they are computationally demanding. This makes RTRL a suitable choice for implementing in the Venturi rig for the flow estimation. Figure 8 shows the experimental results obtained using model-drilling fluid in the test Venturi rig. The flow rate estimation using RNN is compared with the estimation previously made using static ANN and SVR. The comparison shows that RNN has better performance than other empirical models. The MAPE for RNN, ANN and SVR are 5.6\%, 8.5\%, and 7.7\% respectively.

For the future work, we will try to improve the sensor measurements using suitable signal processing. As shown in Figure 4, the output mass flowrate using Coriolis mass flowmeter is less noisy as compared to the three input level measurements. Since the model completely depends on the data, we will work on online signal processing of level sensor measurements to reduce the noise in the measurements. In Figure 7 and Figure 8, we can see discontinuous peaks in the predictions of all the empirical models. By implementing these three models as an integral part of the processing algorithms (signal and control), we believe that 
Table 2. Optimal parameters for different learning algorithms.

\begin{tabular}{lcccc}
\hline Learning algorithms & Epochs & Number of previous inputs & Number of previous outputs & Mape [\%] \\
\hline BPTT & 200 & 1 & 3 & 2.97 \\
RTRL & 500 & 4 & 4 & 2.55 \\
EKF & 20 & 4 & 4 & 3.70 \\
\hline
\end{tabular}
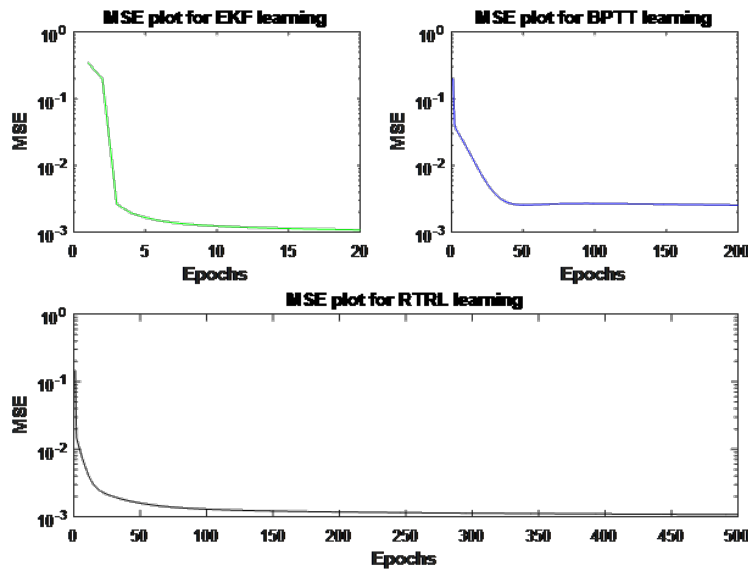

Figure 5. Mean Squared Error (MSE) plot for three different learning algorithms in RNN. Simulation results.
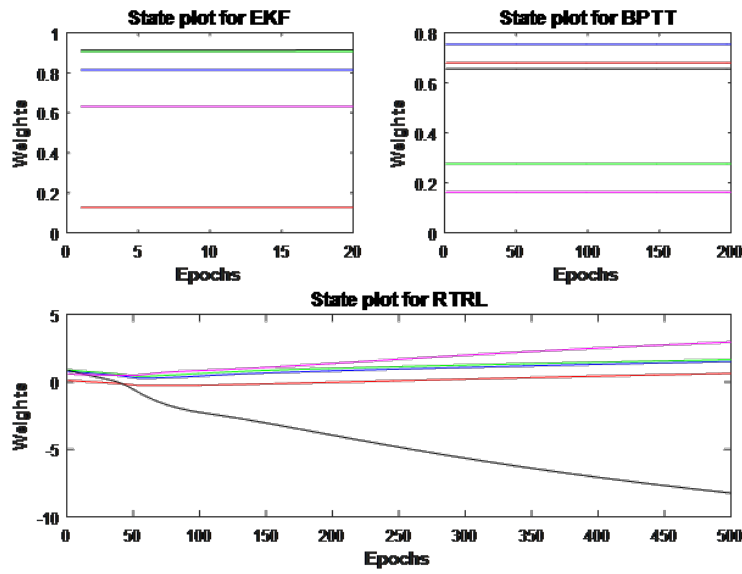

Figure 6. Different weights of the network in a state plot illustrating the convergence of the learning algorithms. Simulation results.

our model can be trained and operated with less noisy data resulting in improved predictions.

\section{Conclusions}

One way of having safe and efficient drilling operation is by continuously monitoring the properties of drilling mud. Any unwanted change in fluid properties can lead to two main problems; the influx of formation fluid and circulation fluid loss. The delta flow measurement while drilling is one of the best methods to detect the early influx or early fluid loss. In this paper, we introduced

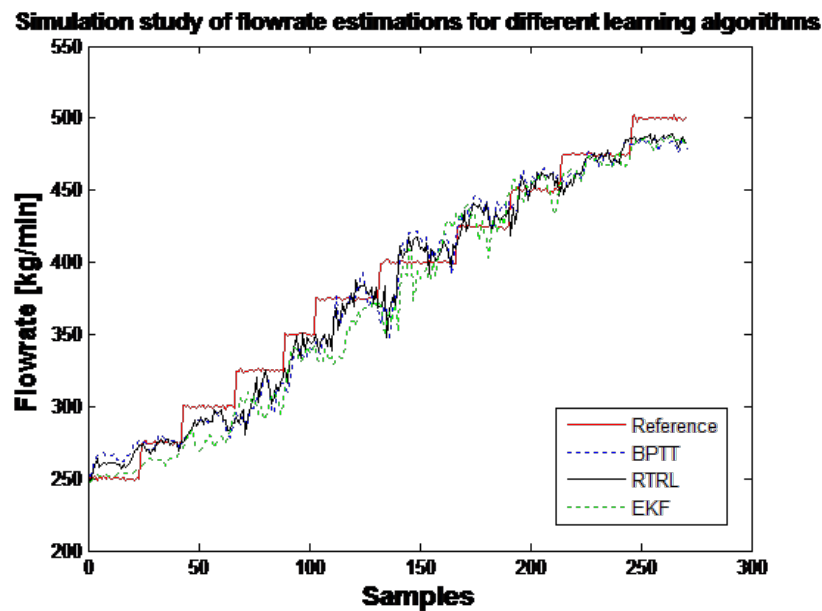

Figure 7. Comparison of flow rate estimation of Recurrent Neural Network (RNN) with three different learning algorithms with respect to Coriolis mass flow measurement as a reference measurement. Simulation results.

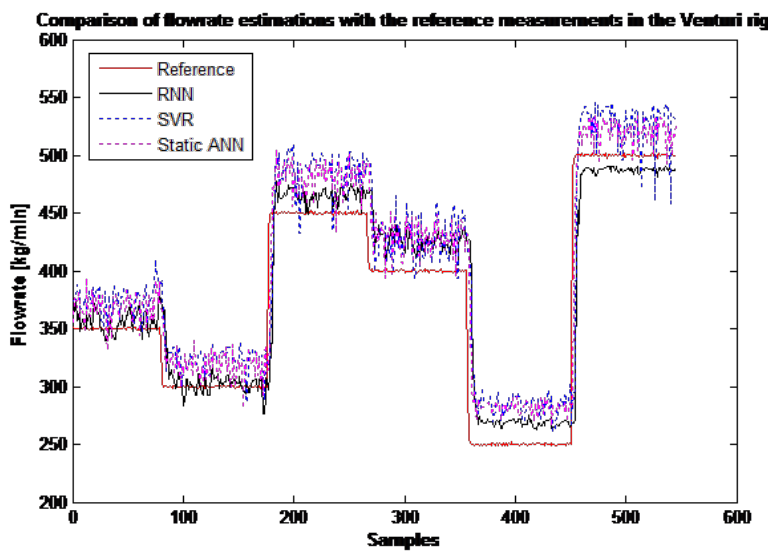

Figure 8. Comparison of flow rate estimations of a Dynamic Artificial Neural Network with Real Time Recurrent Learning algorithm (MAPE of 5.6\%), a static Artificial Neural Network model (MAPE of 8.5\%) and a Support Vector Regression model (MAPE of 7.7\%) with respect to the Coriolis mass flow measurement as a reference flow measurement. Based on experiments using the Venturi-rig.

dynamic Artificial Neural Network to estimate the flow rate of non-Newtonian drilling fluids in an open channel venturi flume, which can be used for outflow measurement while determining delta flow. With Recurrent Neural Network, we simulated three different learning algorithms; Back Propagation Through Time, Real-Time Re- 
current Learning and Extended Kalman Filter algorithm. The simulation results show that BPTT and EKF converge very quickly as compared to RTRL algorithms. Whereas, RTRL algorithm is more accurate, less complex and computationally faster than other two algorithms. So, based on this simulation analysis, RNN with RTRL algorithm is selected for the practical implementation. In the Venturi rig, RNN model with RTRL is implemented along with static ANN and Support Vector Regression (SVR) models. The experimental estimations of flow rates with respect to reference flow rate using Coriolis mass flowmeter show that the estimates based on RNN model has higher accuracy compared to ANN and SVR models. This improved performnce is due to the fact that RNN contains previous inputs and outputs as additional inputs for the current time, which are not considered in static ANN and SVR models. This study shows that non-intrusive ultasonic level measurements of the drilling fluid in an already existing open Venturi channel is a possible alternative to expensive devices such as Coriolis mass flowmeters to measure flow of drilling fluid.

\section{Acknowledgement}

The Ministry of Education and Research of the Norwegian Government is funding Khim Chhantyals $\mathrm{PhD}$ studies at University College of Southeast Norway (USN). Minh Hoang was partly involved in the development of this paper in conjunction with his master thesis at USN in 2016, (Hoang, 2016). The authors at USN appreciate the collaboration with and support from STATOIL for the assembly and commissioning of the open channel Venturi-rig with the various sensors and control system dedicated to the studies related to non-Newtonian fluids. We appreciate the expert advice on drilling operations by Dr. Geir Elseth of STATOIL. In addition, we acknowledge the practical work done by various groups of bachelor and master students of USN in conjunction with this project. Part of the work done is associated with the project SEMI-KIDD funded by the Research Council of Norway.

\section{References}

C. E. Agu and B. Lie. Numerical solution of the saint vernant equation for non-Newtonian fluid. In Proceedings of the 55th Conference on Simulation and Modelling (SIMS 55), Modelling, Simulation and Optimization, 21-22 October 2014, Aalborg, Denmark, number 108, pages 218-228. Linköping University Electronic Press, 2014a.

C. E. Agu and B. Lie. Smart sensors for measuring fluid flow using a venturi channel. In Proceedings of the 55th Conference on Simulation and Modelling (SIMS 55), Modelling, Simulation and Optimization, 21-22 October 2014, Aalborg, Denmark, number 108, pages 229-240. Linköping University Electronic Press, 2014b.

T. H. Ali, S. M. Haberer, I. P. Says, C. C. Ubaru, M. L. Laing, O. Helgesen, M. Liang, and B. Bjelland. Automated alarms for smart flowback fingerprinting and early kick detection. In
SPE/IADC Drilling Conference. Society of Petroleum Engineers, 2013.

C. Berg, A. Tharanga, C. E. Agu, K. Chhantyal, and F. Mohammadi. Simulatioin of open channel flow for mass flow measurement. Technical report, University of South East Norway, Norway, 2013.

M. Boden. A guide to Recurrent Neural Network and backpropagation. Technical report, Halmstad University, Sweden, 2001.

D. B. Budik. A Resource Efficient Localized Recurrent Neural Network Architecture and Learning Algorithm. Technical report, University of Tennessee, USA, 2006.

R. Caenn, C. H. Darley, and G. R. Gray. Composition and properties of drilling and completion fluids. Gulf professional publishing, 2011.

K. Chhantyal, M. Hoang, H. Viumdal, and S. Mylvaganamand. Dynamic artificial neural network (dann) matlab toolbox for time series analysis and prediction. In 9th EUROSIM Congress on Modelling and Simulation, EUROSIM 2016. SIMS Conference on Simulation and Modelling, 2016a.

K. Chhantyal, H. Viumdal, S. Mylvaganam, and G. Elseth. U1trasonic level sensors for flowmetering of non-newtonian fluids in open venturi channels: Using data fusion based on artificial neural network and support vector machines. In Sensors Applications Symposium (SAS), 2016 IEEE, pages 1-6. IEEE, 2016b.

E. O. Dijk. Analysis of Recurrent Neural Networks with application to speaker independent phoneme recognition. Technical report, University Twente, Enschede, The Netherlands, 1999.

F. Frenzel. Industrial flow measurement basics and practice. Technical report, ABB automation products Gmbh, 2011.

G. Geratebau. Equipment for engineering education, intruction manual HM 162.51 Venturi flume. Technical report, Germany, 2013.

S. Hauge and K. Øien. Deepwater horizon: Lessons learned for the Norwegian petroleum industry with focus on technical aspects. Chemical Engineering, 26, 2012.

M. Hoang. Viscosity measurement of non-Newtonian fluids. Technical report, University of South East Norway, Norway, 2016.

M. Kamyab, S. R. Shadizadeh, H. Jazayeri-rad, and N. Dinarvand,. Early kick detection using real time data analysis with dynamic neural network: a case study in Iranian oil fields. In Nigeria Annual International Conference and Exhibition. Society of Petroleum Engineers, 2010.

P. Kim. Kalman filter for beginners: with MATLAB examples. CreateSpace, 2011.

M. W. Mak, K. W. Ku, and Y. L. Lu. On the improvement of the real time recurrent learning algorithm for recurrent neural networks. Neurocomputing, 24(1):13-36, 1999. 


\section{Appendix}

List of symbols and abbreviations

\begin{tabular}{ll}
\hline Symbol & Quantity \\
\hline ANN & Artificial Neural Network \\
BPTT & Back Propagation Through Time \\
CFD & Computational Fluid Dynamics \\
DANN & Dynamic Artificial Neural Network \\
EKF & Extended Kalman Filter \\
$\mathrm{n}$ & Number of folds \\
LT & Level Transmitter \\
MAPE & Mean Absolute Percentage Error \\
MSE & Mean Squared Error \\
N & Number of neurons \\
O & Order \\
$P_{b}$ & Bottom hole pressure \\
$P_{f}$ & Formation pressure \\
$P_{f f}$ & Formation fracture pressure \\
RNN & Recurrent Neural Network \\
RTRL & Real Time Recurrent Learning \\
SVR & Support Vector Regression \\
$\mathrm{t}$ & Time \\
\hline
\end{tabular}

